\title{
HAUSDORFF OPERATORS ON THE WEIGHTED HERZ-TYPE HARDY SPACES
}

\author{
JIANMIAO RUAN AND DASHAN FAN
}

\begin{abstract}
In this paper, we study the high-dimensional Hausdorff operators on the weighted Herz-type Hardy spaces and obtain some substantial extensions from the previous results in [3]. Particularly, for the Hausdorff operators, we establish their sharp boundedness on the power weighted Herz-type Hardy spaces. Our results reveal that the Housdorff operators have better performance in the Herz-type Hardy spaces $H \dot{K}_{q}^{\alpha, p}\left(\mathbf{R}^{n} ; w\right)\left(h \dot{K}_{q}^{\alpha, p}\left(\mathbf{R}^{n} ; w\right)\right)$ than their performance in the Hardy spaces $H^{p}\left(\mathbf{R}^{n} ; w\right)\left(h^{p}\left(\mathbf{R}^{n} ; w\right)\right)$ when $0<p<1$.
\end{abstract}

Mathematics subject classification (2010): 42B30, 42B35. weight.

Keywords and phrases: Hausdorff operator, Herz-type Hardy space, local Herz-type Hardy space,

\section{REFERENCES}

[1] A. Baernstein And E. SAwyer, Embedding and multiplier theorems for $H^{p}\left(\mathbf{R}^{n}\right)$, Mem. Amer. Math. Soc. 53 (1985).

[2] A. Beurling, Construction and analysis of some convolution algebras, Ann. Inst. Fourier (Grenoble) 14 (1964), 1-32.

[3] J. Chen, D. FAn And J. Li, Hausdorff operators on function spaces, Chin. Ann. Math. (Ser. B) 33 (2012), 537-556.

[4] J. ChEn, D. FAn AND S. WANG, Hausdorff operators on Euclidean space (A survey article), Appl. Math. J. Chinese Univ. (Ser. B) 28 (2014), 548-564.

[5] J. Chen, D. FAN AND C. ZHANG, Multilinear Hausdorff operators and their best constants, Acta Math. Sinica (Ser. B) 28 (2012), 1521-1530.

[6] J. ChEn AND X. ZHu, Boundedness of multidimensional Hausdorff operators on $H^{1}\left(\mathbf{R}^{n}\right)$, J. Math. Anal. Appl. 409 (2014), 428-434.

[7] M. Christ AND L. Grafakos, The best constants for two non-convolution inequalities, Proc. Amer. Math. Soc. 123 (1995), 1687-1693.

[8] D. FAN AND X. Lin, Hausdorff operator on real Hardy spaces, Analysis 34 (2014), 319-337.

[9] D. FAN AND D. YANG, The weighted Herz-type Hardy spaces $h \dot{K}_{q}^{\alpha, p}\left(w_{1}, w_{2}\right)$, Approx. Theory Appl. 13 (1997), 19-41.

[10] Z. Fu, L. GRAFAKos, S. LU AND F. ZhaO, Sharp bounds for $m$-linear Hardy and Hilbert operators, Houston J. Math. 38 (2012), 225-243.

[11] Z. FU, Z. LIU, S. LU AND H. WANG, Characterization for commutators of $n$-dimensional fractional Hardy operators, Sci. China Math. (Ser. A) 50 (2007), 1418-1426.

[12] G. GAO, X. Wu AND W. Guo, Some results for Hausdorff operators, Math. Inequal. Appl. 18 (2015), $155-168$.

[13] G. GaO And Y. Zhong, Some inequalities for Hausdorff operators, Math. Inequal. Appl. 17 (2014), 1061-1078.

[14] J. Garcia-Cuerva, Weighted $H^{p}$ spaces, Dissertationes Math. 162 (1979), 1-63.

[15] J. Garcia-Cuerva And M.-J. L. Herrero, A theory of Hardy spaces associated to Herz spaces, Proc. London Math. Soc. 69 (1994), 605-628.

[16] D. Goldberg, A local version of real Hardy spaces, Duke Math. J. 46 (1979), $27-42$. 
[17] L. Grafakos, Modern Fourier Analysis, Second edition, Graduate Texts in Math. 250, Springer, New York, 2009.

[18] C. HerZ, Lipschitz spaces and Bernstein's theorem on absolutely convergent Fourier transforms, J. Math. Mech. 18 (1968), 283-324.

[19] Y. Kanjin, The Hausdorff operator on the real Hardy spaces $H^{p}(\mathbf{R})$, Studia Math. 148 (2001), $37-45$.

[20] M. LEE, Convolution operators on the weighted Herz-type Hardy spaces, J. Approx. Theory 138 (2006), 197-210.

[21] A. LeRner And E. Liflyand, Multidimensional Hausdorff operators on real Hardy spaces, J. Aust. Math. Soc. 83 (2007), 79-86.

[22] E. LiflyAnD, Open problems on Hausdorff operators, Complex Analysis and Potential Theory, World Sci. Publ., Hackensack, NJ, (2007), 280-285.

[23] E. Liflyand, Boundedness of multidimensional Hausdorff operators on $H^{1}\left(\mathbf{R}^{n}\right)$, Acta Sci. Math. (Szeged) 74 (2008), 845-851.

[24] E. LifLYAND, Hausdorff operators on Hardy spaces, Eurasian Math. J. 4 (2013), 101-141.

[25] E. LifLYAND AND A. MIYACHI, Boundedness of the Hausdorff operators in $H^{p}$ spaces, $0<p<1$, Studia Math. 194 (2009), 279-292.

[26] E. LifLYAND AND F. MóRICZ, The Hausdorff operator is bounded on the real Hardy space $H^{1}(\mathbf{R})$, Proc. Amer. Math. Soc. 128 (2000), 1391-1396.

[27] E. LiflyAND AND F. MóRICZ, The multi-parameter Hausdorff operator is bounded on the product Hardy space $H^{11}(\mathbf{R} \times \mathbf{R})$, Analysis 21 (2001), 107-118.

[28] E. LiflyAND AND F. MóRICZ, Commutating relations for Hausdorff operators and Hilbert transforms on real Hardy space, Acta Math. Hungar. 97 (2002), 133-143.

[29] X. Lin And L. Sun, Some estimates on the Hausdorff operator, Acta Sci. Math. (Szeged) 78 (2012), $669-681$.

[30] S. Lu, Herz type spaces, Adv. Math. (China) 33 (2004), 257-272.

[31] S. Lu And D. YANG, The local version of $H^{p}\left(\mathbf{R}^{n}\right)$ spaces at the origin, Studia Math. 116 (1995), $103-131$.

[32] S. LU AND D. YANG, Oscillatory singular integrals on Hardy spaces associated with Herz spaces, Proc. Amer. Math. Soc. 123 (1995), 1695-1701.

[33] S. Lu And D. YAng, The decomposition of weighted Herz space on $\mathbf{R}^{n}$ and its applications, Sci. China Math. (Ser. A) 38 (1995), 147-158.

[34] S. Lu And D. YAng, The weighted Herz-type spaces and its applications, Sci. China Math. (Ser. A) 38 (1995), 662-673.

[35] S. Lu AND D. YANG, Herz-type Sobolev and Bessel potential spaces and their applications, Sci. China Math. (Ser. A) 40 (1997), 113-129.

[36] S. Lu and D. Yang, Some characterizations of weighted Herz-type Hardy spaces and its applications, Acta Math. Sinica (N.S.) 13 (1997), 45-58.

[37] S. Lu, D. YANG AND G. Hu, Herz type spaces and their applications, Science Press, 2008.

[38] A. MiYACHI, Boundedness of the Cesàro operator in Hardy space, J. Fourier Anal. Appl. 10 (2004), 83-92.

[39] F. Móricz, Multivariate Hausdorff operators on the spaces $H^{1}\left(\mathbf{R}^{n}\right)$ and $\operatorname{BMO}\left(\mathbf{R}^{\mathrm{n}}\right)$, Anal. Math. 31 (2005), 31-41.

[40] B. Muckenhoupt, Weighted norm inequalities for the Hardy maximal function, Trans. Amer. Math. Soc. 165 (1972), 207-226.

[41] J. RUAN AND D. FAn, Hausdorff operators on the power weighted Hardy spaces, J. Math. Anal. Appl. 433 (2016), 31-48.

[42] J. O. Strömberg And A. Torchinsky, Weighted Hardy spaces, Lecture Notes in Math. 1381, Springer, Berlin, 1989.

[43] F. WEISZ, The boundedness of the Hausdorff operator on multi-dimensional Hardy spaces, Analysis 24 (2004), 183-195. 